\title{
Pratiques
}

Linguistique, littérature, didactique

187-188| 2020

Enseignement du texte littéraire dans l'espace

francophone: pratiques, formation, recherche

\section{«Ça a bien marché ! » : de la créativité lectorale à la créativité didactique et pédagogique dans la conduite d'une lecture analytique en classe.}

"It worked well!". From readership creativity to didactic-pedagogical creativity

in conducting analytical reading in the classroom.

\section{Bénédicte Shawky-Milcent}

\section{(2) OpenEdition}

Journals

\section{Édition électronique}

URL : https://journals.openedition.org/pratiques/9222

DOI : $10.4000 /$ pratiques.9222

ISSN : 2425-2042

\section{Éditeur}

Centre de recherche sur les médiations (CREM)

\section{Référence électronique}

Bénédicte Shawky-Milcent, « « Ça a bien marché ! » : de la créativité lectorale à la créativité didactique et pédagogique dans la conduite d'une lecture analytique en classe. », Pratiques [En ligne], 187-188 | 2020, mis en ligne le, consulté le 20 juillet 2021. URL : http://journals.openedition.org/pratiques/9222 ; DOI : https://doi.org/10.4000/pratiques.9222

Ce document a été généré automatiquement le 20 juillet 2021.

(c) Tous droits réservés 


\section{«Ça a bien marché !» : de la créativité lectorale à la créativité didactique et pédagogique dans la conduite d'une lecture analytique en classe.}

"It worked well!". From readership creativity to didactic-pedagogical creativity in conducting analytical reading in the classroom.

Bénédicte Shawky-Milcent

\section{Introduction}

1 Un recensement rapide des pistes didactiques partagées par les professeurs de français dans le secondaire - notamment au lycée, par l'exploration de sites dédiés à l'enseignement $d u$ français, de ressources académiques mises en ligne, de manuels scolaires, d'ouvrages de didactique - révèle l'existence de nombreuses propositions de démarches créatives pour précéder, accompagner et prolonger la lecture analytique d'un texte. Ainsi certains enseignants demandent-ils à leurs élèves de se livrer à une réception sensible des extraits étudiés (par le dessin, l'accompagnement musical, etc.). D'autres relient la lecture analytique à la réception d'œuvres empruntées à des domaines autres que la littérature. D'autres encore, pour prolonger une étude du texte, invitent leurs élèves à des réécritures artistiques diverses, en mobilisant le théâtre, le cinéma, la peinture, la photographie, ou encore l'écriture créative. Certaines de ces propositions émanent des travaux sur le sujet lecteur (par exemple Ahr, 2013). Dans ce cas, la réception de l'apprenti lecteur tend à être accueillie dans toutes ses dimensions, intellectuelle, sensorielle, imaginative, affective, axiologique. Les activités proposées complètent, en quelque sorte, l'accès uniquement intellectuel au texte, tel qu'il a prévalu pendant longtemps dans l'histoire de l'explication de texte. D'autres parmi ces 
propositions doivent peut-être au dialogue entre les arts encouragés par l'institution scolaire et la vie culturelle. Toutes ont pour arrière-plan « le retour du sensible » (Virat \& Lenzi 2018) qui a marqué les sciences humaines et sociales au cours des 20 dernières années, ce que d'aucuns ont proposé d'appeler le « tournant émotionnel », à partir de la découverte du rôle joué par les émotions dans les conduites humaines (Damasio, 1999). Je me propose dans cet article d'interroger les raisons et les conditions grâce auxquelles, aux yeux des enseignants qui les conçoivent, certains dispositifs créatifs dans la conduite d'une lecture analytique "marchent bien" auprès des élèves. L'hypothèse suivante a guidé ma réflexion: le succès perçu par l'enseignant d'une démarche créative d'analyse littéraire ne tiendrait-il pas au fait que cette démarche puiserait dans la créativité qui a accompagné sa lecture singulière du texte ? Dans un premier temps, à partir d'une définition générale de la créativité, je rappellerai la nature et le rôle de la créativité lectorale dans la perspective des théoriciens du lecteur empirique et j'analyserai sa place dans les textes officiels pour l'enseignement du français. Dans un deuxième temps, je montrerai que l'enseignant de Lettres concevant une lecture analytique pour sa classe déploie une double créativité : l'une en prise directe avec sa réception personnelle du texte, l'autre en réponse didactique et pédagogique aux besoins de ses élèves, définissant deux champs de réflexion complémentaires à priori distincts. L'analyse du témoignage d'une enseignante me permettra d'amorcer une réflexion sur le dialogue possible entre ces deux créativités. Dans un troisième temps, j'examinerai l'hypothèse d'un continuum entre créativité lectorale et créativité didactique en interrogeant cette fois le sentiment de réussite exprimé par des enseignants à propos de trouvailles créatives mises en œuvre dans leur conduite de la lecture analytique auprès de leurs élèves.

\section{La créativité du lecteur empirique, grande oubliée de l'institution scolaire}

\section{Qu'est-ce que la créativité ?}

2 S'il existe de nombreuses définitions de la créativité, rendant complexe l'analyse de son rôle dans l'apprentissage (Capron Puozzo, 2016, p. 6), toutes s'accordent sur les points suivants.

3 La créativité est la faculté de l'imagination à trouver une solution inédite à un problème donné. Elle se distingue de l'inventivité par le fait qu'elle ne surgit jamais ex nihilo : elle nait d'une difficulté, d'un obstacle, d'une résistance offerts par une réalité, que cette réalité soit psychique ou tangible. Si l'on en croit G. Aznar (2016), la créativité se distingue de la création par le fait qu'elle est motivée par une contrainte externe, contrairement à la création qui répond à une demande interne du sujet. Si la création n'obéit à aucune fonctionnalité, la créativité, quant à elle, a une utilité : elle permet de résoudre un problème. Appelée aussi "pensée magique " par opposition à la pensée logique (Brabandère, 2008), elle procède par analogie, par intuition, par association d'idées, et de ce point de vue, s'oppose à la démarche rationnelle avec laquelle le plus souvent elle compose.

4 Un rapide détour par la psychanalyse est éclairant pour notre propos: selon D. Winnicott, la créativité nait chez l'enfant dans l'espace transitionnel du jeu par lequel l'enfant surmonte la séparation d'avec sa mère. Si «le principe de réalité est une sale 
histoire ", écrit-il, la créativité permet de l'oublier. D. Winnicott (1988 [1986], p. 43) précise : «La créativité, c'est le faire qui dérive de l'être, elle manifeste la vie du sujet, elle est consubstantielle à l'être et au sentiment de soi ».

5 Donc la créativité naîtrait de la contradiction insurmontable entre le désir et son caractère inassouvissable: dans le sillage des travaux de D. Winnicott, R. Diatkine souligne en 1973, lors d'un entretien radiodiffusé avec P. Emmanuel, que la créativité devient une source de plaisir puisqu'elle est cette capacité à se représenter ce qui manque. «La créativité, c'est justement une façon de trouver une solution à ce désir inassouvissable », ajoute-il. Mais il précise que ce qui va importer, c'est la façon dont les adultes vont accueillir la créativité de l'enfant, le plaisir qu'ils vont ressentir, et manifester, au moment où elle s'exprime.

6 À la source de la créativité, il y aurait donc un double plaisir : celui de répondre à un désir et celui de partager cette réponse avec les autres, de tirer un plaisir de leur plaisir. Et si l'on en croit M. Picard (1985), toute écriture et toute lecture réinventent indéfiniment ce jeu originel. La place du désir, du plaisir d'assouvir ce désir et de le partager sont au cœur de la créativité de la lecture et peut-être de la créativité didactique. C'est ce que je vais essayer de montrer à présent.

\section{La créativité du lecteur empirique}

7 J'en viens à la créativité impulsée chez le lecteur par la lecture du texte littéraire. Par rapport à la définition générale proposée ci-dessus, il est important de noter que la créativité est bien une réponse apportée par le lecteur à la résistance que lui oppose le texte. Le lecteur produit mentalement un texte intérieur (Fourtanier, Mazauric \& Langlade, 2011) hétéroclite fait d'impressions diverses et d'inférences imprévisibles par lesquelles il éprouve sa liberté. Par le déploiement de son imaginaire, il rend le texte concret, habitable en quelque sorte. Cette concrétisation complète certes les blancs du texte, selon l'expression de W. Iser (1976), mais elle résulte aussi de la confrontation avec son altérité radicale - comme l'ont rappelé M. Macé (2015) et F. Le Goff (2017).

B. T. Fitch (1991) a très bien décrit le phénomène de concrétisation :

[...] le lecteur transforme par ses facultés imaginatives l'espace représenté dans le texte en espace imaginé, il fait sien ce qui n'existait auparavant, avant le début de sa lecture, que sous une forme latente. D'ailleurs, ce qui est concrétisé revêt une forme qui témoigne déjà de son appartenance au sujet concrétisant, étant littéralement la création de ce dernier, puisque c'est dans les ressources de l'acquis de sa propre expérience que le lecteur puise de quoi concrétiser les référents fictifs.

La concrétisation puise en effet dans tout ce qui constitue le lecteur (expériences, souvenirs, connaissances...). Ainsi, ce dernier habille le texte de visages, d'émotions, de fantasmes, d'idées qui lui appartiennent en propre et le mêle au temps phénoménologique de sa vie. Cette "activité créatrice du lecteur» (Picard, 1989) remplit trois fonctions. Elle est tout d'abord à la source du plaisir de lire et de la jouissance esthétique, comme l'a montré H. R. Jauss (1978, p. 143-144) dans son analyse de l'expérience esthétique: «La conscience en tant qu'activité productrice crée un monde qui est son œuvre propre». La créativité permet au lecteur dans son for intérieur de plier l'œuvre à son usage. Le lecteur, tel un interprète au sens musical du terme, «trouve apparemment dans son jeu un équilibre entre l'exécution scrupuleuse de sa partition et la réalisation de ce qu'il cherche confusément à faire avec la musique ", nous dit M. Picard (1989, p. 89). C'est parce que le lecteur peut se projeter 
dans le texte, s'identifier aux personnages qu'il éprouve du plaisir à lire, et parce que ce monde-là, recomposé par son imaginaire, lui appartient qu'il peut en profiter. C'est sa créativité de lecteur qui lui permet d'activer des scénarios imaginaires, de satisfaire ses désirs de manière détournée par le biais de l'activité fictionnalisante (Langlade, 2006). Par ailleurs, la créativité du lecteur accompagne l'interprétation du texte, joue un rôle décisif dans le processus heuristique (Fitch, 1991). Enfin, elle contribue à laisser une trace dans la mémoire : nos souvenirs de lecture sont aux couleurs de notre imaginaire. Cette créativité, qui se prolonge dans les réinventions de la mémoire du lecteur empirique dessine donc un trait d'union entre le monde du texte et son monde intérieur, et elle devient parfois le tremplin pour une authentique création. M.-A. Murail explique (L'école des loisirs, 2018), par exemple, qu'elle réécrit dans son roman Malo de Lange une scène d'injustice jugée insupportable par la lectrice qu'elle a été de Sans Famille d'H. Malot (1878). Pour toutes ces raisons, les chercheurs en didactique de la littérature ont proposé au cours des dernières années de pratiquer dans le cadre du cours de français des «écritures de la réception » ménageant une place à la créativité des apprentis lecteurs (Le Goff \& Fourtanier, 2017).

\section{La créativité du lecteur empirique dans les textes officiels : circonspection, embarras et ambigüité}

10 Un rapide détour par les Instructions officielles pour l'enseignement du français des 40 dernières années (Shawky-Milcent, 2014) montre qu'à l'égard de cette créativité du lecteur empirique, l'école, quant à elle, ne s'est jamais vraiment affranchie des propos de G. Lanson tenus en 1925 :

On ne songe même pas à condamner la rêverie dont je parlais tout à l'heure, l'activité créatrice de l'esprit du lecteur qui prend le texte seulement comme tremplin pour s'élancer dans les espaces du concevable ou de l'imaginable. Mais il importe de distinguer, dans ce qu'excitent en moi Montesquieu ou Pascal, Racine ou Victor Hugo, ce qui est en moi, de ce qui est en eux. Il faut que j'aie appris à distinguer le sens du livre de l'usage que j'en fais. (Lanson, 1925, p. 38)

11 Le mot " créativité » fait néanmoins une apparition appuyée dans les programmes de 1981, sans doute sous l'influence de l'engouement qu'elle suscite alors dans la réflexion pédagogique (Dosnon, 1996) ${ }^{1}$. Les rédacteurs des programmes en font alors surtout une compétence, que le contact avec les textes littéraires contribuerait à développer. Proposant ainsi, dans la liste des techniques à acquérir par les élèves, des « opérations de créativité ", ils précisent :

Il ne s'agit pas ici de définir des actes de création, qui, dans les domaines littéraire, artistique, scientifique, politique, donnent naissance à des réalisations originales. La pédagogie du français facilite aux élèves l'intelligence des œuvres, mais ne se fixe pas pour mission de leur apprendre à les produire. En revanche, elle s'attache à développer en chacun la capacité d'apporter des solutions autant que possible personnelles aux problèmes précis auxquels il est affronté. À cette fin, elle propose des activités qui associent aux démarches de la raison les ressources de l'imagination et de la sensibilité, font servir l'analyse à la synthèse, favorisent l'esprit d'initiative et le sens de la responsabilité. Dans cette mesure, elle entraine aux opérations de créativité. (IO, 1981, p. 19 ; Shawky-Milcent, 2014)

12 Un peu plus loin dans le texte, ces mêmes rédacteurs, déclinant les différentes formes d'explication de texte écrivent: "Une étude pleinement fidèle est une étude pleinement créatrice " (ibid, p. 27). Mais ce qui pourrait ressembler à une 
reconnaissance de la créativité du lecteur empirique dans l'interprétation se heurte à la mise en valeur du sens (unique) du texte : "Étudier un texte, c'est rendre compte de ce qu'il dit, de ce qu'il suggère, de ce qu'il implique ", est-il précisé (ibid.). Dans les Instructions officielles de 1987, introduisant l'exercice de la lecture méthodique, le terme de créativité disparait complètement. Domine alors une grande prudence à l'égard des réactions personnelles des élèves, réactions qu'il s'agit « d'élucider, de confirmer ou de corriger » (Ministère de l'Éducation nationale, 1987, p. 40). En 2000, le mot créativité est à nouveau absent des programmes. Les écrits d'invention, faisant leur apparition, pourraient être conçus comme un prolongement créatif du texte de lecteur; ils sont en réalité nettement subordonnés à la lecture du texte littéraire : ils " contribuent à une meilleure compréhension des lectures, et permettent aux élèves de construire leur réflexion sur les genres et les registres $»^{2}$. Le terme de créativité ressurgit dans les programmes de $2010^{3}$ dans son acception usuelle de disposition individuelle :

L'acquisition de ces connaissances et de ces capacités va de pair avec des attitudes intellectuelles qui se caractérisent par la curiosité, l'ouverture d'esprit, l'aptitude à l'échange, l'appropriation personnelle des savoirs et la créativité (Ministères de l'Éducation nationale, de la Jeunesse et des Sports, 2010).

13 En effet, la notion est assimilée à une "attitude intellectuelle", favorisée par les connaissances et compétences engrangées au contact des œuvres littéraires. De la créativité du lecteur lors de sa fréquentation du texte littéraire, il ne semble pas être question.

Enfin, dans les derniers programmes de lycée de 2019, le terme est présent dans le Préambule; prenant sans doute en compte certains travaux des didacticiens sur les écritures de la réception, les rédacteurs associent la créativité du lecteur à la mémoire personnelle de la littérature : il s'agit de "structurer cette culture en faisant droit à la sensibilité et à la créativité des élèves ». On observe que les connotations de liberté associées au terme de créativité sont contrebalancées par d'autres approches très rationnelles valorisées dans ce même préambule pour affermir cette culture: "L'acquisition de connaissances solides dans la discipline, notamment dans les domaines de l'analyse littéraire et de l'histoire littéraire "4. Par ailleurs, les "écrits d'appropriation ", qui apparaissent dans ces tout derniers programmes, laissent une place explicite à la réception personnelle des textes par les élèves. Mais le fait qu'ils soient nettement distingués des exercices du commentaire et de la dissertation, semble exclure à priori la créativité du lecteur empirique, telle qu'elle peut s'exprimer plus ou moins librement dans un écrit d'appropriation, de l'acte herméneutique. Ainsi note-ton l'ambigüité persistante de l'institution à l'égard de la créativité du lecteur, reconnue mais reléguée soit à l'espace privé, soit dans les marges des activités scolaires " sérieuses " , quasiment absente des textes officiels (le potentiel émancipateur d'une créativité ouvrant vers la création semblant susciter la méfiance ${ }^{6}$ ), et pourtant encouragée comme levier pédagogique dans les ressources académiques mises par les inspecteurs régionaux à la disposition des enseignants ${ }^{7}$ (où les démarches retenues sont présentées comme des exemples stimulants).

\section{De la créativité lectorale à la créativité didactique}

En quoi consisterait la créativité de l'enseignant proposant à sa classe l'analyse d'un texte littéraire? Quels liens peut-on tisser entre sa lecture personnelle, souvent secrète 
et évanescente, et la construction d'une séance pour la classe, professionnelle et partagée, en prise avec la réalité de la classe?

L'enseignant, ayant provisoirement vaincu dans son travail de préparation les résistances que lui opposait le texte, se heurte à l'obstacle suivant, pouvant se décliner en de multiples sous-obstacles : la difficulté, pour toutes les raisons que l'on connait, à faire entrer un groupe d'adolescents dans un texte littéraire. On peut admettre que toute démarche rompant avec le déroulement convenu et ritualisé de l'analyse - recueil des impressions de lecture/création d'un débat interprétatif/élaboration d'axes de lecture/mise au jour d'une interprétation du texte - est potentiellement créative, toute démarche qui s'adapte aux besoins spécifiques d'un groupe singulier, dans une situation pédagogique donnée. Cette fois, contrairement à la créativité du lecteur, il y a, de la part de l'enseignant, une action exercée de manière tangible sur une réalité concrète, par le recours par exemple à des objets : le calque, le carnet, la feuille de dessin, la photographie. Par la modification de la configuration spatiale ou temporelle de la séance... Par des activités renouvelant les pratiques habituelles : carnet à quatre mains/journal du personnage/théâtre des lectures/journal dialogué/feuille d'ambiance poétique/carte postale du lecteur/carte heuristique... Par la mobilisation d'autres pratiques artistiques, etc. Soulignons que la créativité n'est pas l'apanage de l'enseignant qui partagerait la vision de la lecture littéraire défendue par les didacticiens de la littérature comme va-et-vient dialectique entre participation et distanciation (Dufays, Gemenne \& Ledur, 1996). On peut imaginer un enseignant qui aurait une conception textualiste de l'enseignement de la littérature, et qui ferait preuve de créativité pour atteindre ses objectifs. Donc, il existe potentiellement tout un éventail d'interventions créatives allant d'un geste professionnel ponctuel à une multicréativité. Par exemple, dans son roman Un jour par la forêt, M. Sizun présente une enseignante en difficulté, qui tente de remédier à l'hostilité de ses élèves, provoquée par une explication formaliste du poème :

Combien, dans la classe, se sont vraiment intéressés à Demain dès l'aube? Honnêtement combien? [...] Et si elle essayait de leur rendre la poésie plus proche avant de faire appel à ce que le garçon appelait « tout ce bazar »? Si elle tentait de leur parler avec des mots simples, en faisant d'abord appel à leur sensibilité ? Et si, au lieu de leur lire le texte elle-même - elle sait bien qu'elle n'est pas formée à ça, et qu'elle a, oui, on le lui a dit, une voix déplaisante - elle leur faisait entendre l'enregistrement d'un bon comédien? (Sizun, 2013, p. 260)

17 À l'opposé, on peut évoquer la démarche multi-créative adoptée par Mme L., enseignante dans un lycée du Pas-de-Calais, rencontrée en formation, dont le travail sur Réparer les vivants de M. de Kérangal, est accessible en ligne. Ressentant la résistance de ses élèves à entrer dans la littérarité de ce roman paru en 2013, l'enseignante imagine un dispositif d'écriture collaborative dans les marges, en filant la métaphore de la greffe d'organes, au cœur de l'intrigue (Lobry, 2018). Les lectures analytiques programmées trouvent chacune leur place dans ce projet d'envergure qui, par ses différentes facettes, répond à toute une série de difficultés.

Difficulté à entrer dans le texte? Les élèves écrivent entre les lignes, dialoguent avec lui. Difficulté des élèves à s'intéresser à un extrait imposé par le professeur? Ils choisissent eux-mêmes les passages qui feront l'objet d'une lecture analytique. Difficulté pour les petits parleurs à participer au débat interprétatif ? Les adolescents travaillent en groupes, par blogs interposés. Difficulté à percevoir la littérarité du texte? Les lycéens aboutiront à une version réinventée du roman, enrichie et 
collaborative. Difficulté à accompagner chacun dans le projet? Par le recours à des outils informatiques et aux ressources offertes par le numérique, l'enseignante procure à chaque élève une aide personnalisée. On voit bien ici que Mme L. ne répond pas seulement aux difficultés globales de la transmission de la littérature ou aux prescriptions institutionnelles. Elle répond aussi à l'hétérogénéité et au profil de cette classe de première scientifique précise (par une collaboration avec le professeur de SVT et l'infirmière) $)^{8}$.

Mais alors, quel serait le rapport entre la créativité privée de l'enseignant lecteur et la créativité didactique mise en œuvre en classe, offerte au jugement et soumises à des critères d'efficacité objectivables? Mme L. accepte d'évoquer, dans un entretien par email ${ }^{9}$, sa réception personnelle du roman de $\mathrm{M}$. de Kérangal. Elle explique à quel point cette œuvre l'a bouleversée, et en même temps apaisée :

Je me souviens avoir été bouleversée par ma lecture, complètement happée par le souffle des phrases, séduite par l'alliance entre la description d'une réalité médicale dépeinte très précisément et son dépassement par le romanesque, l'épique [...]. Je ne me le suis pas dit comme cela au moment de ma lecture, mais je pense enfin que ce roman a éveillé en moi des souvenirs [...], il me réparait en quelque sorte [...]. obscurément et sans pouvoir le formuler clairement, je pense qu'il y avait en moi un besoin très marqué de réparation, une angoisse sourde.

C'est ce qu'elle voudrait faire vivre aux élèves, souhaitant partager avec eux cette expérience de comblement que lui a offert ce texte littéraire :

En le refermant, j'ai tout de suite pensé à l'étudier avec mes élèves, en me disant que le rythme trépidant qui semble inspiré des séries, les questions abordées les concerneraient et seraient à même de les toucher.

L'enseignante prend alors conscience de son texte intérieur au moment de la lecture, des points de suture qu'elle a fait mentalement pour le rendre lisible :

J'avais été frappée par l'écriture par fragments lors de la lecture qui laisse la place à l'imagination du lecteur, un peu comme une machine qui a besoin du lecteur pour fonctionner.

La conception pour ses élèves du dispositif de prélèvement des extraits étudiés en lecture analytique s'impose à elle quand elle songe aux émotions très fortes qu'elle a pu ressentir :

Mon premier souci était de ne pas les «choquer »: je ne voulais pas leur imposer d'étudier des scènes dont j'avais peur qu'elles ne les troublent, et ils ont fini par choisir eux-mêmes des moments très durs. Inversement, ils n'ont pas retenu des moments qui m'avaient plu et qui m'auraient attirée (la rencontre entre Simon et Juliette, ou le moment où Thomas Rémige répare le corps de Simon par exemple). C'est de là que m'est venue l'idée de leur faire « prélever » eux-mêmes les textes.

Pour cautériser les blessures que le texte, éprouvant, pourrait créer sur ses élèves, elle leur propose d'écrire entre les lignes (ce que l'on fait toujours quand on lit, on écrit dans sa tête entre les lignes, surtout quand le texte est rude) ; ainsi, elle matérialise ce qu'elle a fait mentalement; elle rend concrète pour ses élèves la concrétisation à laquelle elle s'est livrée :

Ensuite, je ne sais pas dire exactement ce qui m'a conduite à leur proposer d'intervenir dans le roman pour l'augmenter, plusieurs facteurs [...].

Je crois que du prélèvement, en filant la métaphore je suis arrivée à cette idée de greffe en m'inspirant de l'histoire du livre lui-même [...]. Il me semblait que les élèves pouvaient être bouleversés eux aussi par cette histoire, et que greffer leurs écrits étaient comme une façon de les réparer en tant que lecteurs (je ne sais pas si je suis très claire), en s'appropriant le texte pour le faire évoluer dans la direction 
qu'ils souhaitaient. Beaucoup de mes élèves ont des vies très heurtées, compliquées

et accidentées, je me suis dit que ce roman pouvait leur apporter quelque chose. conduisent un enseignant à considérer qu'une lecture analytique créative menée en classe est réussie. Je postule que c'est parce que un enseignant est relié, sans en être forcément conscient, à la créativité de sa réception personnelle du texte littéraire qu'un tel dispositif créatif fonctionne, selon sa perception subjective, mais sans doute aussi selon des critères de réussite plus objectifs (comme l'implication des élèves dans les tâches demandées par exemple ou leur mémoire des activités menées). Plus généralement, je postule que le professeur créatif en matière de lecture littéraire est celui qui, non seulement laisse une place silencieuse à sa réception personnelle du texte proposé aux élèves, mais aussi à ses pratiques de lecture privée, à sa façon de lire, et à la conscience qu'il a, par expérience, du processus phénoménologique de la lecture. C'est la raison pour laquelle une démarche originale, sensible, imaginative pour l'étude d'un texte ou d'une œuvre est difficilement transposable à l'identique, et ne peut être prescrite. Pour vérifier cette hypothèse, je m'appuierai provisoirement sur une préenquête que j'ai menée en 2018 auprès de 50 enseignants de français rencontrés dans ma pratique professionnelle ${ }^{10}$, en vue d'une recherche plus ample sur l'enseignant lecteur(Shawky-Milcent, 2018).

Voici un aperçu de l'échantillon consulté :

Tableau 1. Synthèse de l'échantillon consulté (enquête 2018)

\begin{tabular}{|l|l|}
\hline $\begin{array}{l}50 \text { questionnaires envoyés } \\
\text { par courriel }\end{array}$ & 36 réponses reçues, 14 absences de réponse \\
\hline Sexe & 6 hommes, 30 femmes \\
\hline Établissement d'exercice & $\begin{array}{l}20 \text { en lycée (dont } 2 \text { privés sous contrat), } 12 \text { en collège (dont } 2 \text { en } \\
\text { REP+), } 2 \text { en LP, } 1 \text { cité scolaire, } 1 \text { professeur indépendant }\end{array}$ \\
\hline Diplôme & $\begin{array}{l}18 \text { certifiés, } 15 \text { agrégés, } 2 \text { professeurs de lycée professionnel, } \\
1 \text { titulaire d'une maîtrise }\end{array}$ \\
\hline Âge & $\begin{array}{l}\text { entre } 20 \text { et } 30 \text { ans }: 4 \text { entre } 31 \text { et } 40 \text { ans : } 10 \text { entre } 41 \text { et } 50 \text { ans : } 13 \\
\text { entre } 51 \text { et } 60 \text { ans }: 6 \text { Plus de } 60 \text { ans : } 3\end{array}$ \\
\hline Aire géographique & Hauts-de-France $: 20$ Île-de-France : 9 Rhône-Alpes : 7 \\
\hline
\end{tabular}


Dans ce questionnaire visant à explorer les liens entre pratiques de lecture privées et pratiques professionnelles figurait notamment la question :

Quand vous étudiez un extrait d'œuvre littéraire avec vos élèves, prenez-vous appui

- parmi d'autres propositions - sur votre approche personnelle du texte?

Il est intéressant de remarquer que tous les enseignants sollicités affirment s'appuyer sur leur approche personnelle du texte pour l'étudier avec leurs élèves : "toujours » pour $25 / 36$, « assez régulièrement » pour $10 / 36$, «parfois » pour $1 / 36$. Un peu plus loin, leur étaient posées les trois questions suivantes :

Pourriez-vous citer au moins un dispositif pédagogique qui a été particulièrement efficace auprès de vos élèves pour leur faire découvrir un texte ou une œuvre?

Dans quelle mesure ce dispositif a-t-il un lien avec votre lecture personnelle de ce texte ou de cette œuvre?

Ce dispositif a-t-il un lien avec votre goût pour d'autres formes artistiques, peinture, cinéma, musique, ou autre?

Concernant ces liens entre démarche perçue comme réussie et lecture personnelle, voici les résultats obtenus :

Tableau 2. Enquête 2018.

\begin{tabular}{|l|l|}
\hline Non aux trois questions ou absence de réponse & $4 / 36$ \\
\hline Non mais 1 exemple & $2 / 36$ \\
\hline Oui sans justification & $\mathbf{4 / 3 6}$ \\
\hline Oui avec justification & $\mathbf{2 4 / 3 6}$ \\
\hline
\end{tabular}

Ces résultats provisoires et notamment les 28 réponses positives obtenues sur 36 personnes interrogées tendent à valider mon hypothèse. On a rappelé que la créativité du lecteur est suscitée par trois aspects de la lecture: elle surgit de la confrontation avec les obstacles présentés par le texte, de la transformation du texte à son usage pour en éprouver du plaisir, et de la création d'un nouveau texte intérieur, perceptible dans le souvenir de lecture, qui pourra devenir le tremplin d'une véritable œuvre. Ces trois aspects essentiels de la lecture se retrouvent nettement dans les réponses obtenues : dans la variation créative qu'il introduit dans son cours, qu'elle soit minime ou de grande ampleur, l'enseignant écoute une part de ses tâtonnements, de son appropriation subjective du texte, et du potentiel de réécriture qu'il a intériorisé en lisant cet extrait ou cette œuvre littéraire. Je me propose d'analyser quelques exemples représentatifs de ces trois tendances dans cet échantillon.

\section{La créativité didactique surgie du souvenir de la résistance offerte par le texte}

Quand l'enseignant re-parcourt en pensée non seulement son interprétation du texte mais également la résistance qu'il a éprouvée à tel ou tel endroit et qu'il offre en partage à ses élèves, il ouvre des zones d'indétermination qui deviennent également des espaces de dialogue. Je prendrai l'exemple de Mme P., jeune enseignante qui évoque l'étude de la Fortune des Rougon d'É. Zola (1871) en seconde et décrit la démarche suivante : 
Afin de commencer la lecture de La Fortune des Rougon (et pour vérifier également la compréhension du chapitre I), j'ai organisé une séance qui s'intéressait en priorité aux espaces dans le roman. Consignes données :

Sur une feuille A3, faire un croquis le plus complet possible de la ville de Plassans en replaçant :

- Les lieux importants (qui ont été identifiés dans la première phase de discussion) : Quartier Saint-Marc, Vieux Quartier, Ville Neuve, Aire Saint-Mittre, Jas-Meiffren

- Les routes et espaces naturels qui délimitent la ville : Route de Nice, Route de Lyon, Rue de la Banne, Cours Sauvaire, La Viorne, Collines des Garrigues

- Les personnages ou catégories de personnages associés à chaque lieu

- Enfin les symboliques propres à chaque espace

Cette séance s'avère très fructueuse :

La séance a été extrêmement productive car les élèves étaient surpris du travail de dessin/croquis qui leur était demandé - la forme était finalement très libre. Poser les espaces réels et symboliques du premier chapitre a permis à beaucoup de clarifier leur compréhension de la ville mais aussi des enjeux romanesques liés à la situation géographique. Certains ont même continué leur travail à la maison pour rendre un croquis très complet montrant qu'ils ont cherché à imaginer les lieux décrits. Cela a permis à d'autres de mieux aborder la suite de la lecture.

Ce dispositif a-t-il un lien avec la lecture personnelle de ce texte ou de cette œuvre? Remontant à sa propre lecture, l'enseignante souligne :

Lors de la première lecture du roman, j'ai éprouvé la nécessité de tracer sur papier un plan de la ville car le premier chapitre est extrêmement riche en termes topographiques. J'ai constaté que ce simple croquis a beaucoup guidé ma lecture et j'ai continué à le développer au fur et à mesure des chapitres avec les symboliques, les déplacements des personnages, ou encore des citations associées à chaque espace. Cela a abouti à un document très personnel sur ma compréhension de l'œuvre. J'ai tenté de m'appuyer sur cette pratique personnelle pour la transformer en activité pédagogique.

Ce dispositif a-t-il un lien avec son gout pour d'autres formes artistiques, peinture, cinéma, musique, ou autre?

J'aime beaucoup dessiner et peindre. J'ai aussi une mémoire plutôt visuelle et ai besoin de représenter dans l'espace certains éléments. C'est peut-être cela qui a orienté mes choix lors de cette séance.

On peut relier cet exemple au recours à l'image qui s'est répandu pour amorcer une interprétation du texte (Ahr, 2013). Proposer à des élèves de trouver une image pour faire écho à une lecture personnelle, c'est en quelque sorte matérialiser leur concrétisation imageante et les rendre sensibles aux amorces d'interprétations recelées par le choix de telle ou telle représentation iconographique. C'est ce que fait Mme R. par exemple en demandant à ses élèves de produire une illustration de l'incipit de $L a$ Mort du roi Tsongor de L. Gaudé (2002). Cette proposition fait écho à sa lecture personnelle du texte, car, note-t-elle :

À la lecture de ce roman, j'avais été fascinée par la peinture de cet orient mythique et je crois que j'avais envie que les élèves voyagent aussi et donnent leur propre interprétation du lieu. mythique ", laissant entrevoir sa propre rêverie intérieure. 


\section{La créativité didactique surgie du désir de revivre le plaisir éprouvé lors de la lecture et de le partager}

Partager le plaisir de la lecture, c'est le réitérer, et on touche là l'essence de la transmission, et du bonheur d'enseigner. On pourrait prendre plusieurs exemples dans le panel de réponses, dans lesquels on voit qu'une idée nait du souvenir de la rencontre intime avec un personnage, du halo affectif déposé dans la mémoire par une scène ou par une description.

Mme R, professeur au collège, imagine adresser une lettre à chacun de ses élèves :

Cette année en $5^{\mathrm{e}}$, nous avons étudié L'île au trésor de Stevenson. J'ai d'abord écrit une lettre fictive en employant un lexique propre aux pirates de la part de Long John Silver que j'ai déposé sur leur table avant qu'ils ne rentrent en classe. En résumé, cette lettre les invitait à l'aventure à bord de l'Hispaniola et à se renseigner sur la piraterie avant d'embarquer pour en comprendre les dangers.

Cette lettre avait pour objectif de les plonger dans le thème de la séquence d'y être "invité», tout en leur indiquant le prochain travail à effectuer avant de commencer l'étude de l'œuvre: des exposés sur le monde des pirates. J'ai eu l'impression qu'ils se sentaient investis d'une mission, et qu'elle était importante pour poursuivre l'aventure.

Et elle précise que c'est l'attachement au personnage qui a guidé son choix :

J'ai particulièrement apprécié ce personnage ambivalent qu'est Long John Silver. J'ai alors eu l'idée de la lettre pour établir un lien complice, comme celui qu'il entretient avec Jim Hawkins. Non seulement elle servait d'accroche, mais elle permettait aussi de révéler le caractère du personnage mais aussi sa façon d'agir avec les autres.

On peut citer encore Mme B., qui raconte :

J'ai à l'occasion de l'étude de Notre-Dame de Paris de V. Hugo, créé un power point qui mêlait extraits du texte, anecdotes sur la cathédrale et Histoire. [...] Aujourd'hui, si c'était à refaire je placerais également des extraits de films et comédies musicales.

Faire dessiner la façade principale de la cathédrale à partir de la description les avait aidés aussi.

41 Elle accomplit ce choix avec, précise-t-elle, « l'envie que les élèves ressentent le même plaisir et la même émotion et qu'ils reconnaissent le travail et le génie de l'auteur ».

On peut évoquer cette autre enseignante encore qui, ayant conservé une mémoire auditive du roman de M. Fermine, Neige (1999) propose d'analyser l'incipit à partir d'une écoute des bruits d'une gare au Japon.

\section{La créativité didactique surgie de l'expérience très intime de la lecture, d'une manière singulière de lire...}

C'est le troisième aspect qui émerge dans les réponses à ces questions. Ainsi en est-il de Mme M., qui demande souvent à ses élèves en amont d'une lecture analytique :

Une présentation «scénographiée » accompagnée d'objets, d'odeurs, de musique... à savoir une présentation qui laisse transparaitre le lecteur, qui nous engage à la découverte, dans la pénombre ou les yeux fermés.

Et d'ajouter :

Lorsque je présente une œuvre, je me présente lectrice de ce texte afin d'inviter mes élèves à rejoindre ce cercle de lecteurs, cette communauté. 
Ou encore de Mme F. pour qui les ouvertures proposées à la suite d'une lecture analytique sont celles qui correspondent à ses goûts :

Si je travaille les réécritures du mythe d'Orphée, je prendrai le temps de leur faire écouter Monteverdi car c'est une musique que j'apprécie; si je travaille Rimbaud je leur lirai des extraits de Michon et leur ferai découvrir Ernest Pignon-Ernest.

Dès lors, cette créativité réveille le potentiel de réécriture offert par l'œuvre et dans lequel l'imaginaire du professeur a pu s'engouffrer. On ne sera pas étonné que parmi les dispositifs créatifs proposés, les enseignants évoquent de nombreux travaux de réécriture. Ainsi, ce qui apparait dans ces exemples, ressentis comme des réussites pédagogiques, c'est la manière dont l'enseignant va puiser dans l'autre moitié du lecteur, celle que la lecture analytique traditionnelle laisse dans l'ombre: dans sa sensibilité et son imaginaire. La lecture analytique ne se réduit alors plus à la mise au jour d'un contenu, à la transmission d'un savoir objectivable, elle est aussi initiation à des gestes de lecture que l'élève pourra reproduire avec d'autres textes.

Ce qui crée peut-être l'accueil favorable des élèves, souligné dans les témoignages recueillis, c'est que l'écoute de sa propre lecture par l'enseignant l'engage à écouter avec une attention accrue la créativité de l'élève. Ainsi en est-il dans cet exemple présenté dans Vers un enseignement de la lecture littéraire au lycée (Ahr, 2013, p. 96), où une enseignante étudie avec une classe La Princesse de Clèves de Madame de La Fayette (1678), et notamment la mort de Mme de Chartres:

En demandant aux élèves de composer à la maison un collage à partir de ce texte, après leur avoir montré quelques créations de Prévert.

Puis, elle analyse ainsi la séance qui suit :

Un détail a retenu l'attention du professeur quand il a pris connaissance des productions personnelles [...]. Cet effet de double entre les deux personnages, la mère et la fille, l'une se reflétant dans l'autre alors qu'elles sont aux deux bouts de la vie a traversé la galerie personnelle du professeur qui a convoqué la référence aux trois âges de la vie. Les tableaux de Baldung et de Klimt ont de nouveau permis de questionner le texte en classe [...] les attitudes de la vieille femme différentes dans les deux tableaux [...] ont été de nouveaux points d'accroche au texte de Mme de Lafayette (Ahr, 2013, p. 97)

\section{Conclusion}

4 Le petit nombre de témoignages cités limite la portée de l'analyse proposée ici ; toute prudence gardée, ces exemples encouragent néanmoins à laisser une place plus nette lors de la formation des enseignants à l'apprentissage d'une créativité réfléchie, qui passerait par la connaissance de soi comme lecteur, par l'écoute des trajets secrets qui conduisent, par tout un jeu de hasards et de coïncidences, aux textes littéraires partagés avec les élèves.

50 Ce serait peut-être une piste pour faire évoluer les pratiques de lecture littéraire, transformer l'exercice figé de la lecture analytique (qui n'est plus envisagé en tant que tel depuis les programmes de 2019), en véritable expérience (Cambron \& Langlade, 2015), permettant à l'élève de vivre toute la créativité de sa lecture de littérature.

51 Il existe actuellement une injonction à la créativité, qui envahit comme un mot d'ordre un peu creux la sphère économique, le monde de l'entreprise, la psychologie, et aussi la pédagogie ${ }^{11}$, comme une issue incertaine à la routine et à la rigidité de certains cadres. 
La créativité est associée à des mots comme stratégie, compétences, efficacité, innovation... dans une course un peu vaine à la performance à tout prix. On pourrait, à l'inverse, montrer combien la créativité du lecteur, partagée grâce à l'acte pédagogique, favorise la liberté intérieure: rêverie offerte par l'altérité du texte, tremplin pour une compréhension de soi et des autres, ouverture vers la création.

\section{BIBLIOGRAPHIE}

AHR, S. (dir.) (2013). Vers un enseignement de la lecture littéraire au lycée, Expérimentations et réflexions. Grenoble : Scéren.

Altet, M. (2001). « Les compétences de l'enseignant professionnel : entre savoirs, schèmes d'action et adaptation, le savoir analyser ». In : Paquet L. et al. (dirs). Former des enseignants professionnels, quelles stratégies? Quelles compétences?. Louvain-la-Neuve : De Boeck.

AzNAR, G. (2016). La créativité : définitions. Paris : Créa Université. En ligne : https://jpp.iut.fr/wpcontent/uploads/2016/04/La-CREATIVITE-DEFINITIONS-Guy-Aznar.pdf.

BRABANDÈRE, L. (de) (2008). Pensée magique, pensée logique. Paris : Editions Le Pommier.

CAPRon Puozzo, I. (2016). « Créativité et apprentissage, dilemme et harmonie ». Revue française de pédagogie 197, p. 5-12. En ligne : https://journals.openedition.org/rfp/5130.

Cambron., M. \& Langlade, G. (dirs) (2015). L'Évènement de lecture. Montréal : Nota Bene.

DAMASIO, A. R, (1999). Le Sentiment même de soi. Corps, émotions, conscience. Trad de l'anglais (États-

Unis) par C. Larsonneur et C. Tiercelin. Paris : O. Jacob

Diatkine, R. \& emmanuel, P. (1973). « La créativité et les âges de l'homme ». Dialogues, France

Culture. Emission du 23 janv. 1973 avec P. Emmanuel et R. Diatkine. En ligne : https://youtu.be/ Mc9K47s-Z7g.

Dosnon, O. (1996). « Imaginaire et créativité, éléments pour un bilan critique ». Pratiques 89, p. 5-24. En ligne : https://www.persee.fr/doc/prati_0338-2389_1996_num_89_1_1765.

DufAYS, J.-L., GEMENNE, L \& LEDUR, D. (1996). Pour une lecture littéraire. Louvain-la-Neuve : De BoeckDuculot

FiтcH, B. T., (1991). « Introduction. Référence, concrétisation, appropriation ». Texte. Revue de critique et de théorie littéraire 11, p. 5-18. En ligne : http://french.chass.utoronto.ca/unsorted/ litera/Revue_Texte/Int11.pdf.

HAMELINE, D., (1973). «La créativité, fortune d'un concept ou concept de fortune ? ». Orientations 47, p. 3-23.

ISER., W. (1976). L'Acte de lecture. Théorie de l'effet esthétique. Trad. De l'allemand par E. Sznycer. Sprimont : P. Mardaga.

JAuss, H.-R. (1978) [1972]. Pour une esthétique de la réception. Trad. De l'allemand par C. Maillard. Paris : Gallimard. 
LANGLADE, G. (2006). « L'activité fictionnalisante du lecteur ». In : Louichon, B. \& Laville, B. (dirs). Les Enseignements de la fiction. Pessac : Presses universitaires de Bordeaux, p. 163-177.

LANSON, G. (1925). «Quelques mots sur l'explication de textes ». In : Méthodes de l'histoire littéraire. Paris : Les Belles Lettres.

LE GofF, F. \& FourTANIER, M.-J. (dirs) (2017). Les Formes plurielles des écritures de la réception. Volume I: Genres, espaces et formes. Vol. 2, Affects et temporalités, Namur : Presses universitaires.

LE GoFF, F. (2017). La Reconnaissance du processus de subjectivation littéraire : un enjeu de la formation initiale des professeurs de français. Dossier pour HDR : Université de Montpellier.

LOBRY, M. (2018). « PNF : ATELIER 5. Comment faire dialoguer des œuvres de statut différent? Littérature de jeunesse, littérature classique et littérature contemporaine ». Compte rendu du huitième Rendez-vous des Lettres. Amiens : Académie d'Amiens. En ligne : http://lettres.acamiens.fr/IMG/pdf/doc_3_presentation_reparer_les_vivants_pnf_.pdf.

MARC, P., (1977). « Une réflexion psychosociologique sur la faveur rencontrée par la notion de créativité ». Bulletin Société Alfred Binet Théodore Simon 77 (558), p. 236-261.

MAzauric, C., Fourtanier, M.-J. \& LANGlade, G., (dirs) (2011). Le Texte du lecteur. Textes de lecteur en formation. 2 vol. Bruxelles : P. Lang.

Ministère de l'Éducation nationale (1987). « La lecture méthodique ». Bulletin officiel de l'Éducation nationale du 5 février 1987. En ligne : http://secoursdefrancais.free.fr/college/documents/htm/ admin_txt_bo.htm\#4.

Ministère de l'Éducation Nationale, de l'Enseignement supérieur et de la Recherche (2001). « Programme d'enseignement du français en classe de première des séries générales et technologiques ». Bulletin Officiel no 28 du 12 juillet 2001. En ligne : https://www.education.gouv.fr/ bo/2001/28/encartc.htm.

Ministère de l'Éducation Nationale, de la Jeunesse et des Sports (2010). « Programme de l'enseignement commun de français en classe de seconde générale et technologique et en classe de première des séries générales et programme de l'enseignement de littérature en classe de première littéraire ». Bulletin Officiel spécial $n^{\circ} 9$ du 30 septembre 2010. En ligne : https:// www.education.gouv.fr/bo/2010/special09/mene1019760a.htm.

Ministère de l'Éducation Nationale, de la Jeunesse et des Sports (2019). « Les programmes du lycée général et technologique ». Bulletin Officiel du 4 avril 2019. En ligne : https:// www.education.gouv.fr/bo/2010/special09/mene1019760a.htm.

PICARD M. (1989). Lire le temps. Paris : Éditions de Minuit.

MACÉ M. (2015). «Questions de lecture, entre expérience et appropriations ». Fabula-LhT. Littérature, histoire, théorie 14. En ligne : http://www.fabula.org/lht/14/mace.html.

L'école des loisirs (2018). « Marie-Aude Murail, auteure de Malo de Lange, fils de voleur ». Vidéo de présentation. Youtube. En ligne : https://youtu.be/7Pula93mxLs.

SIZUN, M. (2013). Un jour par la forêt. Paris : Arléa.

SHAWKY-MiLCENT, B. (2014). L'Appropriation des œeuvres littéraires en classe de seconde. Thèse de doctorat : Université Grenoble Alpes. En ligne : https://tel.archives-ouvertes.fr/tel-01677062. 
SHAWKY-MilCENT, B. (2018). « Comment les professeurs choisissent-ils les textes? ». Le $8^{e}$ rendezvous des Lettres. Bibliothèque nationale de France, 14-15 mai 2018. En ligne : https://www.canalu.tv/video/eduscol/comment_les_professeurs_choisissent_ils_les_textes_au_lycee.44977.

VIRAT, M., LENZI C., (2018). « La place des émotions dans le travail socio-éducatif », Sociétés et jeunesses en difficulté 20. En ligne : https://www.researchgate.net/publication/ 328248200_La_place_des_emotions_dans_le_travail_socio-educatif.

WinNicotT, D. (1988) [1986]. Conversations ordinaires. Trad. De l'anglais par B. Bost. Paris : Gallimard.

\section{ANNEXES}

\section{Grandes lignes de l'enquête menée auprès des enseignants.}

\section{A. Votre profil.}

Items proposés : âge, sexe, parcours universitaire, nombre d'années d'enseignement, statut, établissement fréquenté au moment de l'enquête, classes en charge, missions de formation éventuelles.

\section{B. Votre rapport à la lecture.}

Items proposés : loisirs pratiqués, gout pour la lecture, gout pour l'écriture, histoire personnelle avec la lecture, évènements marquants ayant éventuellement infléchi cette histoire en bien ou en mal, rôle de la lecture privée dans la vie actuelle, œuvres lues au moment de l'enquête, avis sur ces dernières, genres littéraires appréciés, auteurs préférés, références incontournables de la «bibliothèque intérieure ", évènements de lecture, modalités de choix des lectures personnelles, relectures, lieux évoquant la lecture, sensations, sentiments ou émotions associés à la lecture privée.

\section{Votre rapport à votre métier et aux textes littéraires que vous enseignez.}

Items proposés : place des sensations, émotions, sentiments précédemment évoqués dans la pratique professionnelle, choix d'exercer le métier de professeur de Lettres, préférence pour « professeur de Lettres » ou " professeur de français », rôle déterminant joué par un enseignant au cours de la scolarité, si oui influence éventuelle de cet enseignant sur pratique actuelle, objectif prioritaire auprès des élèves, jugement à propos de l'enseignement de la littérature, modalités de choix des lectures cursives proposées aux élèves, exemples de conseils prodigués à une classe pour conseiller un ouvrage, modalités de choix des œuvres étudiées intégralement, modalités de choix des extraits étudiés en classe.

Questions concernant plus particulièrement la lecture analytique : 
Quand vous étudiez un extrait d'œuvre littéraire avec vos élèves, sur quoi prenezvous appui pour préparer votre cours?

Une critique lue dans une revue professionnelle ou grand public?

Toujours-assez régulièrement-parfois-Jamais-Sans opinion

Une étude que vous avez faite à l'université ou au cours d'un stage?

Toujours -Assez régulièrement -Parfois- Jamais- Sans opinion

Un manuel ? Le manuel de la classe?

Toujours - Assez régulièrement- Parfois- Jamais- Sans opinion

Des ressources offertes par internet?

Toujours- Assez régulièrement- Parfois- Jamais- Sans opinion

Votre approche personnelle de ce texte? Si oui, pouvez-vous développer ce point?

Toujours -Assez régulièrement -Parfois- Jamais- Sans opinion

C.10 En six lignes maximum, définissez ce que serait pour vous un cours réussi sur un texte ou sur une œuvre.

C.11 Pourriez-vous citer au moins une démarche pédagogique qui a été

particulièrement efficace auprès de vos élèves pour leur faire découvrir un texte ou une œuvre?

Dans quelle mesure cette démarche a-t-elle un lien avec votre lecture personnelle de ce texte ou de cette œuvre?

Cette démarche a-t-elle un lien avec votre gout pour d'autres formes artistiques, peinture, cinéma, musique, ou autre?

C.12 La chercheuse Annie Rouxel propose de définir ainsi l'identité littéraire :

«La notion d'identité littéraire suppose donc une sorte d'équivalence entre soi et des textes : textes que j'aime, qui me représentent, qui métaphoriquement parlent de moi, qui m'ont fait ce que je suis, qui disent ce que je voudrais dire, qui m'ont révélé à moi-même. Cette notion requiert et établit la mémoire de textes qui ont jalonné un parcours - elle évoque un univers littéraire - mais elle inclut aussi un rapport à la langue, à l'écriture, à la singularité d'une manière de lire... ${ }^{\text { }}$ "

Diriez-vous que votre identité littéraire:

- Coïncide avec votre identité professionnelle?

- Est distincte de votre identité professionnelle?

- Se conjugue avec votre identité professionnelle?

- Autre réponse?

Commentaires éventuels :

\section{Les effets de votre enseignement sur votre relation personnelle à la lecture.}

Items proposés : évolution de l'intérêt porté aux œuvres à la suite de l'étude menée en classe auprès des élèves, rôle joué par le dialogue avec les élèves à propos de tel ou tel texte. 


\section{E. La place tenue par la formation continue dans votre relation à la lecture et dans votre enseignement de la littérature.}

Items proposés : rôle éventuel joué par la formation continue dans la relation à la lecture et dans l'enseignement de la littérature, facteurs d'évolution des pratiques professionnelles

\section{F. Et pour finir....}

Items proposés : jugement porté sur l'intérêt du questionnaire et remarques libres.

\section{NOTES}

1. «La créativité est devenue le pivot d'un système de croyances éducatives qui revendique la spontanéité et qui dénonce l'action sclérosante et étouffante de l'école (Hameline, 1973 ; Marc, 1977)»

2. Ministère de l'Éducation Nationale et du ministère de la Recherche (2001).

3. Ministère de l'Éducation Nationale, de la Jeunesse et des Sports (2010).

4. Ministère de l'Éducation Nationale, de la Jeunesse et des Sports (2019).

5. Les « écrits d'appropriation » rendus quasiment impossibles à réaliser du fait de la lourdeur des programmes.

6. On retrouve cette ambivalence dans tout le système scolaire, la créativité pouvant être tout à la fois valorisée et mise « en tension avec la conception de l'élève idéal [...] associée au non conformisme et aux comportements perturbateurs » (Capron Puozzo, 2016, p. 6).

7. Par exemple le site de l'Académie de Lille : http://lettres.discipline.ac-lille.fr/.

8. Passant ainsi de la didactique à la pédagogie, si l'on se réfère à la distinction établie par M. Altet (2001), entre la didactique qui selon elle est « de l'ordre de la diachronie, du temps fictif de l'anticipation des contenus ", et la pédagogie située dans «le temps synchronique de l'enseignement, pendant le temps réel de l'intervention », "dans la régulation interactive des évènements en classe ».

9. Je remercie très chaleureusement Mme L. pour son témoignage passionnant.

10. Les grandes lignes de ce questionnaire figurent en annexe.

11. Par exemple, 100 idées de stratégies, modèles, postures éducatives pour stimuler sa créativité pédagogique Weber $\mathrm{H}$., article consultable http://www.cedip.developpementdurable.gouv.fr/100-idees-pour-stimuler-sa-creativite-pedagogique-a1322.html

a. Rouxel, A. (2004). « Autobiographie de lecteur et identité littéraire ». In : Langlade G. \& Rouxel A. (dirs.) Le sujet lecteur, lecture subjective et enseignement de la littérature. PUR, coll. Didact français, p.139. 


\section{RÉSUMÉS}

On observe, au collège comme au lycée, des propositions de démarches créatives pour précéder, accompagner et prolonger la lecture analytique d'un texte, encourageant une réception sensible et sensorielle du texte, la mobilisation d'autres formes artistiques, des actualisations variées. Dans cet article nous formons l'hypothèse que la créativité déployée dans ces dispositifs puise dans la créativité lectorale singulière des enseignants ou des enseignants chercheurs qui les conçoivent. Dans un premier temps on interrogera la notion de créativité dans la perspective retenue, puis on confrontera cette hypothèse aux résultats obtenus lors d'une enquête menée auprès de 40 enseignants du secondaire portant sur les liens entre lecture privée et pratique professionnelle.

In middle and high school, there are proposals for creative approaches to precede, accompany and lengthen the analytical reading of a text, encouraging a sensitive and sensory reception of the passage, the mobilization of other artistic forms, and a variety of updates. In this article we postulate that the creativity deployed in these methods relies on the singular readership creativity of the teachers or teacher-researchers who design them.

First, the notion of creativity will be questioned from the chosen perspective, then this hypothesis will be compared with the results of a survey conducted among forty secondary school teachers on the links between private reading and professional practice.

\section{INDEX}

Mots-clés : créativité, texte du lecteur, enseignant lecteur, concrétisation, lecture analytique

Keywords : creativity, reader's text, reader teacher, concretization, reading analytical.

\section{AUTEUR}

\section{BÉNÉDICTE SHAWKY-MILCENT}

Université Grenoble Alpes, Litt\&Arts CNRS, F-38400 Saint-Martin-d'Hères, France 